\title{
Time series of ground reaction forces following a single leg drop jump landing in elite youth soccer players consist of four distinct phases
}

\author{
Duncan P. Fransz ${ }^{\mathrm{a}, \mathrm{b}, *}$, Arnold Huurnink ${ }^{\mathrm{a}, \mathrm{c}}$, Vosse A. de Boode ${ }^{\mathrm{d}}$, Idsart Kingma ${ }^{\mathrm{a}}$, \\ Jaap H. van Dieën ${ }^{a}$ \\ a MOVE Research Institute Amsterdam, Department of Human Movement Sciences, Vrije Universiteit Amsterdam, Amsterdam, The Netherlands \\ ${ }^{\mathrm{b}}$ Department of Orthopaedic Surgery, Zuyderland Medical Center, Heerlen, The Netherlands \\ ${ }^{\mathrm{c}}$ Department of Nuclear Medicine, Academic Medical Center, Amsterdam, The Netherlands \\ d adidas miCoach Performance Centre, AFC Ajax, Amsterdam, The Netherlands
}

\section{A R T I C L E I N F O}

Article history:

Received 11 December 2015

Received in revised form 12 August 2016

Accepted 1 September 2016

\section{Keywords:}

Force plate

Onsite testing

Football

Postural control

Parameter selection

Motor control

\section{A B S T R A C T}

The single leg drop jump landing test may assess dynamic and static balance abilities in different phases of the landing. However objective definitions of different phases following landing and associated reliability are lacking.

Therefore, we determined the existence of possible distinct phases of single leg drop jump landing on a force plate in 82 elite youth soccer players. Three outcome measures were calculated over moving windows of five sizes: center of pressure (COP) speed, COP sway and horizontal ground reaction force (GRF).

Per outcome measure, a Factor Analysis was employed with all windows as input variables. It showed that four factors (patterns of variance) largely ( $>75 \%$ ) explained the variance across subjects/trials along the $12 \mathrm{~s}$ time series. Each factor was highly associated with a distinct phase of the time series signal: dynamic (0.4-2.7 s), late dynamic (2.5-5.0 s), static $1(5.0-8.3 \mathrm{~s})$ and static $2(8.1-11.7 \mathrm{~s})$.

Intra-class correlations (ICC) between trials were lower for the dynamic phases $(0.45-0.68)$ than for the static phases (0.60-0.86). The COP speed showed higher ICC's (0.63-0.86) than COP sway (0.45-0.61) and GRF (0.57-0.71) for all four phases.

In conclusion, following a drop jump landing unique information is available in four distinct phases. The COP speed is most reliable, with higher reliability in the static phases compared to the dynamic phases. Future studies should assess the sensitivity of information from dynamic, late dynamic and static phases.

(c) 2016 Elsevier B.V. All rights reserved.

\section{Introduction}

Single leg balance performance has been significantly related to functional performance [1-3] and injuries, such as ankle sprains [4] and anterior cruciate ligament (ACL) deficiency [5]. However, it has been suggested that single leg jump landing tasks may better detect differences in sensorimotor function than static single leg stance, since they are more challenging and sport specific [6-8]. A jump landing test is a dynamic task where subjects jump either from a box or to a certain height, land upon a force plate on one foot, and stabilize as quickly as possible [9].

\footnotetext{
* Corresponding author at: MOVE Research Institute Amsterdam, Department of Human Movement Sciences, Van der Boechorststraat 9, 1081 BT Amsterdam, The Netherlands.

E-mail address: dpfransz@gmail.com (D.P. Fransz).
}

The most commonly applied outcome measures to quantify performance of the jump landing task are the 'time to stabilization' (TTS) and the 'dynamic postural stability index' (DPSI). The TTS aims to quantify the transition from an instable situation to a stable situation. Large differences exist in TTS calculation methods, therefore studies should be interpreted and compared with caution [10,11]. Calculation of the DPSI is straightforward and quantifies the fluctuation of the resultant ground reaction forces (GRF) around the origin (mean value) for $3 \mathrm{~s}$ following impact [12]. Since impact forces are high, DPSI emphasizes more the landing rather than the stabilizing phase [13]. Both TTS and DPSI have shown higher outcome values for subjects with chronic ankle instability [14-16], or with ACL reconstruction [17,18], compared to healthy controls. However, their applicability with regard to injury risk and monitoring of rehabilitation still needs to be elucidated $[6,13,19,20]$. 
Center of pressure (COP) derivatives, such as 'COP speed' and 'COP sway', have been shown to be highly reliable and valid in single leg balance performance $[15,21,22]$. The COP speed has been able to discriminate between subjects with functional ankle instability and healthy controls [15]. Moreover, COP speed was significantly larger for subjects with chronic ankle instability, than for copers and healthy controls [22]. Surprisingly, however, TTS and DPSI based on jump landing tests appeared to be uncorrelated with these COP derivatives of static single leg stance $[8,23,24]$. Moreover, no correlations were found between static and dynamic performance using the same outcome measures, i.e. 'COP speed' or 'SD of GRF' $[8,25]$. Therefore, one could suggest that static and dynamic tests reflect different aspects of total body sensorimotor function, implying an expanded perspective with regard to injury risk prediction, preventive actions and rehabilitation management.

Moreover, depending on the calculation method, TTS targets different time frames of the GRF following landing. This resulted in large variation in outcome values $(0.5-6 \mathrm{~s})$ and low correlations between calculation methods applied to the same measurement $[10,11]$. Therefore, distinct information may be available within the dynamic phases as well. An interesting characteristic of the jump landing task is that the landing itself, the stabilizing phase and static balance performance can be evaluated [26]. To date, a thorough and systematic evaluation of the complete COP and GRF time series following landing has not been addressed. Such an assessment will give insights in the information available in the data collected in a jump landing task, and will reveal which time frames best reflect this information. In order to facilitate the sensitivity, it is important to optimize the precision of the outcome measure (i.e. reliability or reproducibility). Both the starting point and window size applied to calculate the outcome measures may affect the reliability.

Therefore, the aim of the current study was to determine (1) the existence of possible distinct phases following a single leg drop jump landing task by means of Factor Analysis, (2) the effect of window selection on the reliability of mean COP speed, mean absolute COP sway, and mean absolute horizontal GRF, and (3) the correlation between these outcome measures.

\section{Methods}

\subsection{Participants}

The current data set was acquired at the youth academy of AFC Ajax at the start of the 2013-2014 season. We included the data of 82 players between 11 and 18 years old (mean $\pm S D$; age $14.10 \pm 1.86$ years; height $1.68 \pm 0.12 \mathrm{~m}$; body weight $56.70 \pm 13.20 \mathrm{~kg}$ ), for whom six valid trials were available. At the time of measurements, all players were fit to perform at the highest standard of competitive soccer matches. The local ethics committee approved the research protocol and all players or parents/guardians (depending on the age of the participant) were informed in advance of the procedures involved in the testing program and provided written informed consent.

\subsection{Instrumentation}

Ground reaction forces (GRF) were recorded at 1000 samples/s, using a $40 \times 60 \mathrm{~cm}$ AMTI force plate (type BP400600HF, Advanced Medical Technologies Inc., Watertown, MA, USA). The center of pressure (COP) calculations were based on vertical and horizontal forces in accordance with the manufacturer's manual.

\subsection{Procedures}

The players were asked to jump from an aerobic step of $20 \mathrm{~cm}$ height, which was placed $5 \mathrm{~cm}$ posterior to the force plate, located at $4 \mathrm{~m}$ from the wall. Players took off by means of a small jump with two feet, landed on the testing leg at the center of the force plate, and stabilized as quickly as possible. They had to balance for $15 \mathrm{~s}$ with their hands on their hips, whilst keeping all other movement to a minimum (Fig. 1). Before actual testing commenced, all players completed the regular warm-up, as accustomed before a training session, and performed one practice trial per leg. Both legs were tested thrice; the left leg was appointed the initial testing leg. All trials were performed without shoes. A trial was considered invalid if a player touched the floor with the contralateral leg or if arm movement was used to regain balance.

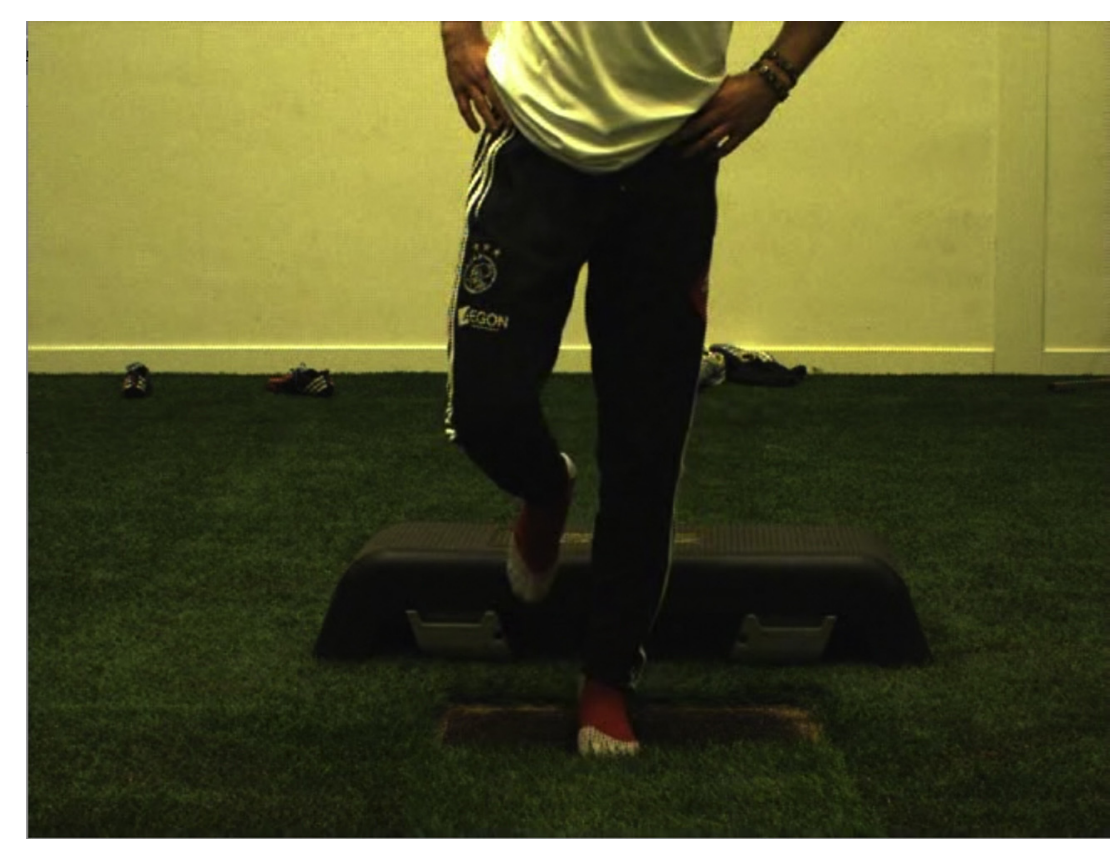

Fig. 1. The experimental setup showing one of the players during the stance phase following the single leg drop jump landing. 


\subsection{Data processing}

A custom MATLAB for Mac (The Mathworks Inc., version R2014a, Natick, RI, USA) program was written for data processing. Data were cropped from time of impact (vertical GRF > 10 N) to $12 \mathrm{~s}$ post-impact and low pass filtered at $12 \mathrm{~Hz}$ with a bidirectional second order Butterworth filter [27].

\subsection{Data analysis}

For each trial, three outcome measures were calculated:

(1) The mean COP speed ('COP speed'), which is the total COP path length divided by trial time [2,28-30].

(2) The mean absolute COP sway ('COP sway'), which is the mean absolute distance of the COP trajectory to the average COP position [3,31].

(3) The mean absolute horizontal GRF ('Hor GRF'), which is the mean length of the GRF vector in the horizontal plane $[15,29]$.

We varied the window size of the moving window over which the outcome measures were calculated $(0.5,1.0,2.0,3.0$, and $5.0 \mathrm{~s})$. For Factor Analyses, the window was moved along the time series from 0 to $12 \mathrm{~s}$ with $0.1 \mathrm{~s}$ per step, resulting in a total of 485 windows. For reliability analyses, windows were moved with $0.001 \mathrm{~s}$ per step. This was done for each trial (3), for each leg (2), for each outcome measure (3) and for each window size (5).
The three outcome measures showed a comparable non normal distribution for all the calculated windows, particularly showing a low and long tail to the right in the histograms.

\subsection{Statistical analysis}

Both limbs were grouped together, resulting in six trials per player, assuming that postural stability is predominately an indicator of whole body sensorimotor function [4].

\subsubsection{Factor analysis}

To examine which of the windows held unique information, we employed a Factor Analysis in IBM SPSS Statistics for Mac (IBM Corp., version 21.0, Armonk, NY, USA). The extraction method was set as principal components. This statistical technique is an elegant procedure to elucidate patterns of co-variation between the input variables (i.e. outcome measures for each window) across the subjects and trials. Each pattern of co-variation is called a 'factor' whereby the first factor explains as much of the variance in the data as possible, the second factor for the residual variance and so on for the subsequent factors. The constraint is that each factor is orthogonal to the preceding factors, ensuring that each factor holds unique information [32].

The input variables for the Factor Analysis were the outcome values calculated for all window sizes $(0.5,1.0,2.0,3.0$ and $5.0 \mathrm{~s})$ and for all starting points between $0 \mathrm{~s}$ and $12 \mathrm{~s}$. All input variables contained the outcome values for each trial (82 players $\times 3$
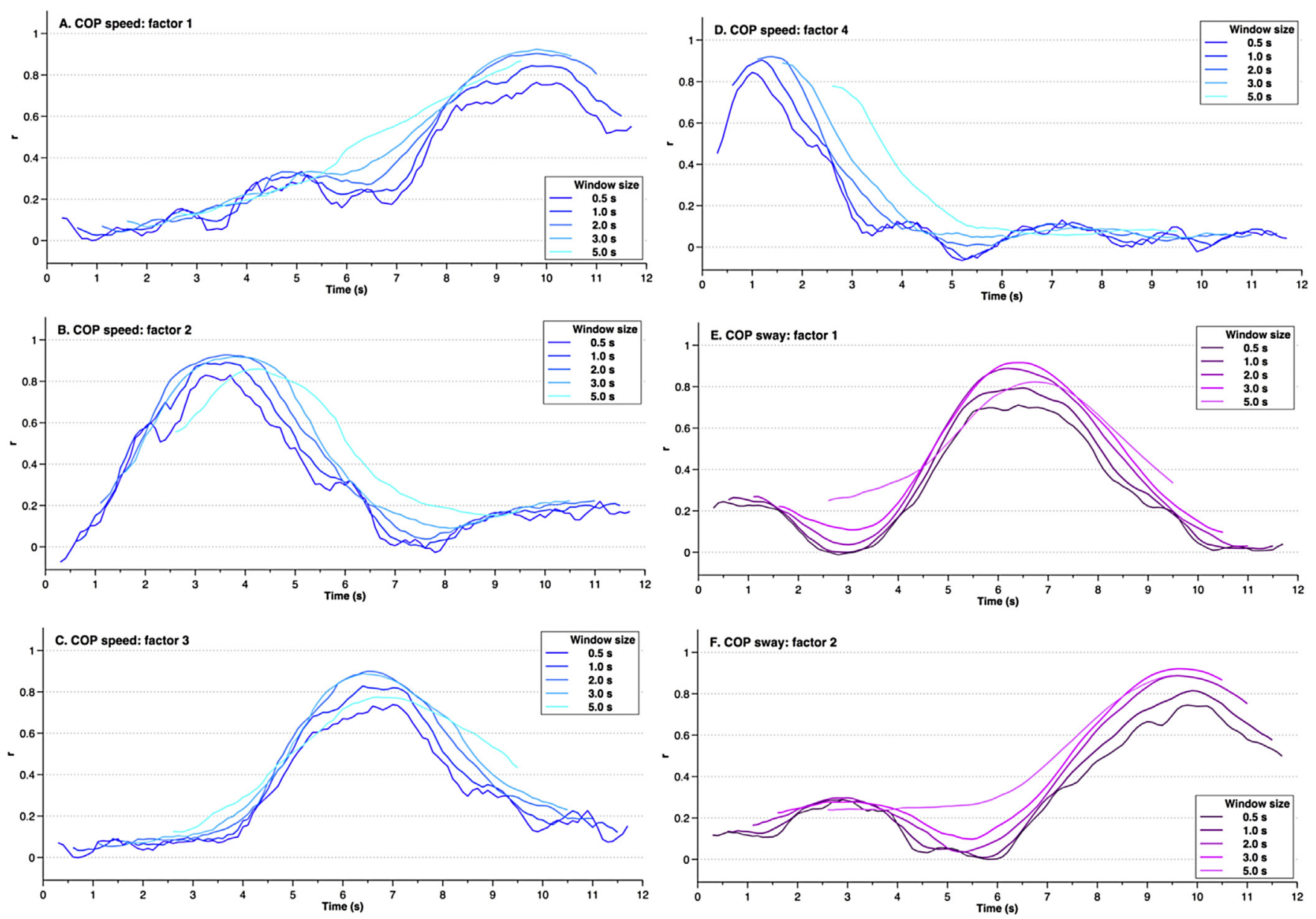

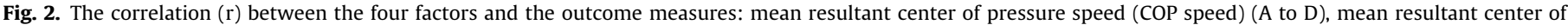

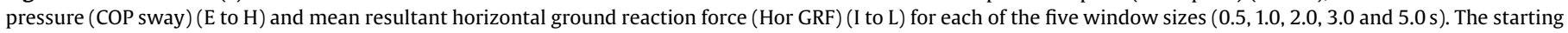
point for each window size is half the length of the window, in order to facilitate comparison. 
trials $\times 2$ legs). The Factor Analysis was performed for each outcome measure (COP speed, COP sway and Hor GRF) separately.

The first step of the Factor Analysis was to assess the appropriate number of factors. A cumulative explained variance above $75 \%$ was set as cut off. The second step of the Factor Analysis was the orthogonal ('varimax') rotation of the data, resulting in a so called 'rotation matrix'. This matrix holds the correlation between each factor and each input variable (i.e. outcome measure for each window).

This allowed the identification of groups of windows that were strongly associated with the factors; hence phases in the time series could be determined. The window with the highest correlation with the factor was considered as the most valid window to represent the distinct phase. In addition and as confirmation, the inter-correlation between the identified windows (i.e. phases) was assessed for the three outcome measures.

\subsubsection{Reliability analysis}

An absolute agreement two-way random model (average measures) was applied to calculate intra-class correlation coefficients (ICC) in order to assess the reliability over trials ( 6 trials per subject) for each outcome measure in each individual window [33].

\subsubsection{Correlation between outcome measures}

Across all trials, inter-item Pearson correlations were calculated between the three outcome measures: COP speed, COP sway and Hor GRF. Possible redundancy between outcome measures was considered if the coefficient of correlation was above 0.70 .
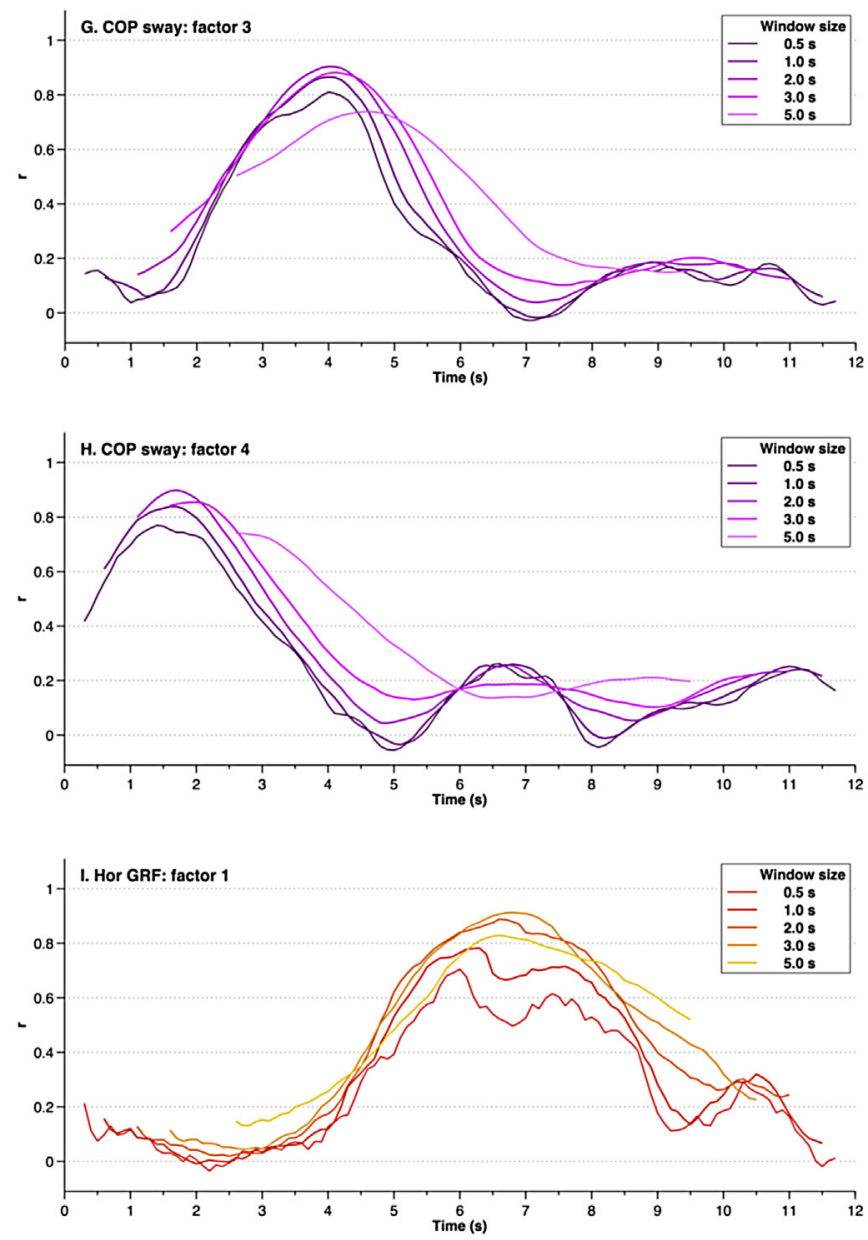

\section{Results}

\subsection{Factor analysis}

The first part of the Factor Analysis showed that the data were to a large extent explained by four factors. For the COP speed, COP sway and Hor GRF these first four factors explained 79.8\%, 77.3\% and $75.3 \%$ of the total variance, respectively. A possible fifth factor would have added only $3.2 \%, 4.8 \%$ and $3.0 \%$, respectively (see Supplementary data 1 ), hence the 'rotation matrix' was built with four factors. Fig. 2 illustrates the 'rotation matrix' as it shows the correlation $(r)$ between the factor and the outcome measures for each window. Each factor showed a consistent and distinct high correlation for a specific range of windows. This indicates the existence of four distinct phases in time, each containing unique information. This was consistent for all window sizes and outcome measures (COP speed, COP sway and Hor GRF). The four factors will henceforward be referred to as representing the four phases: dynamic phase (DP) (Fig. 2d, h and l), late dynamic phase (LDP) (Figs. 2b, g and j), static phase 1 (SP1) (Figs. 2c, e and i) and static phase 2 (SP2) (Figs. 2a, f and k). Furthermore, within each phase, the outcomes of the window sizes of 2 or $3 \mathrm{~s}$ showed the strongest association with the factor. For each factor and outcome measure, we selected the window with the highest correlation with that factor to represent that factor. These highest correlations were always $>0.90$ (Table 1 ). To illustrate the independence of outcome values between phases, we calculated the correlations of outcomes between the windows best representing the four factors. These correlations were all positive, ranging from 0.19 to 0.57 (Table 2).
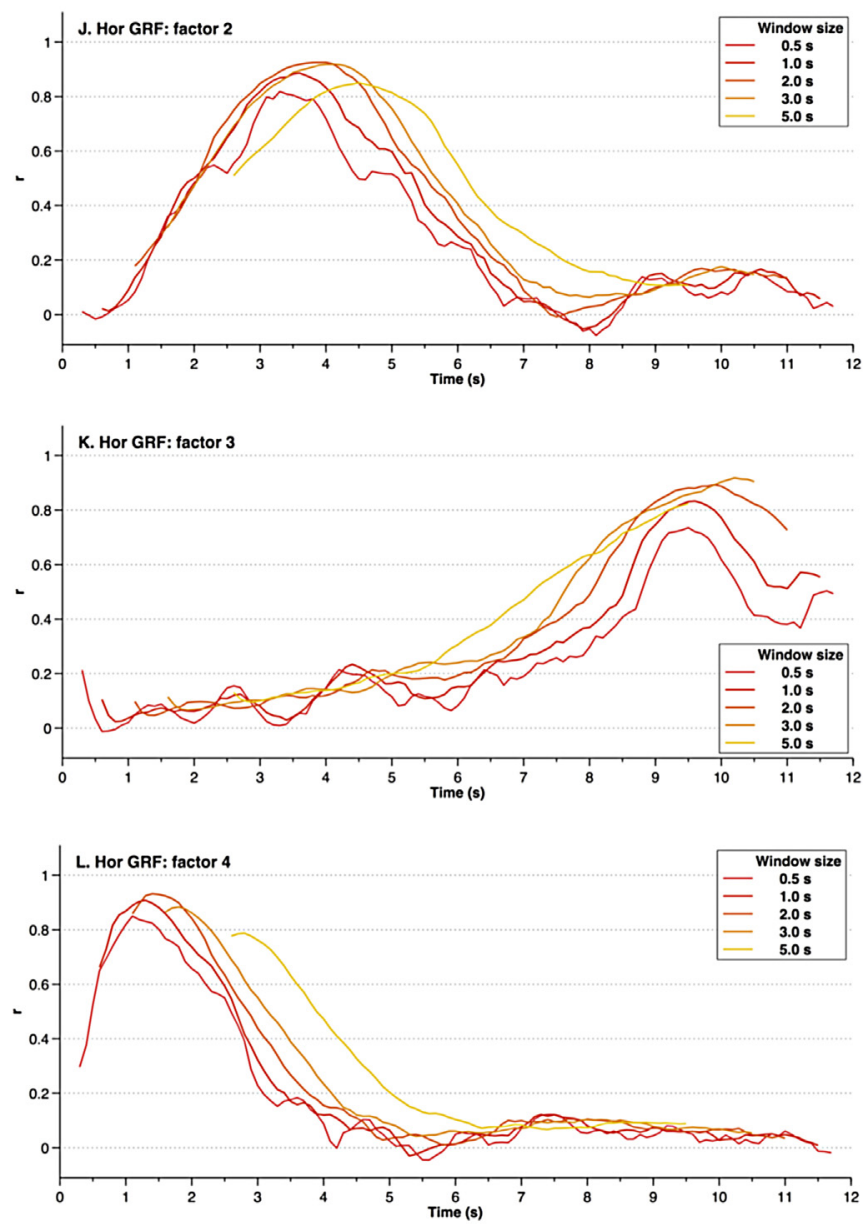

Fig. 2. (Continued) 
Table 1

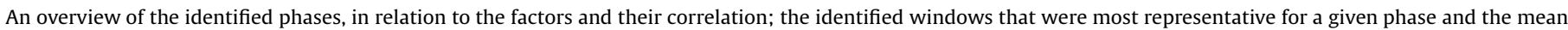

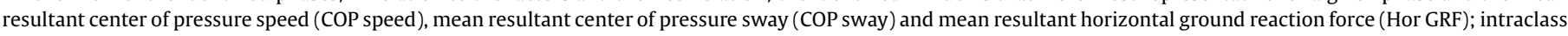
correlation coefficients (ICC) are shown with 95\% confidence interval (95\% ci).

\begin{tabular}{|c|c|c|c|c|c|}
\hline & & Dynamic phase & Late dynamic phase & Static phase 1 & Static phase 2 \\
\hline \multirow[t]{5}{*}{ COP speed $(\mathrm{mm} / \mathrm{s})$} & factor & 4 & 2 & 3 & 1 \\
\hline & correlation & 0.92 & 0.93 & 0.90 & 0.92 \\
\hline & window & $0.4-2.4 \mathrm{~s}$ & $2.6-4.6 s$ & $5.5-7.5 \mathrm{~s}$ & $8.3-11.3 \mathrm{~s}$ \\
\hline & mean $\pm \mathrm{SD}$ & $130.9 \pm 71.6$ & $60.9 \pm 33.8$ & $49.3 \pm 18.3$ & $47.3 \pm 15.5$ \\
\hline & ICC $[95 \% \mathrm{ci}]$ & $0.63[0.49-0.74]$ & $0.68[0.55-0.77]$ & $0.77[0.68-0.84]$ & $0.86[0.81-0.90]$ \\
\hline \multirow[t]{5}{*}{ COP sway (mm) } & factor & 4 & 3 & 1 & 2 \\
\hline & correlation & 0.90 & 0.90 & 0.92 & 0.92 \\
\hline & window & $0.7-2.7 \mathrm{~s}$ & $3.0-5.0 \mathrm{~s}$ & $5.0-8.0 \mathrm{~s}$ & $8.1-11.1 \mathrm{~s}$ \\
\hline & mean $\pm \mathrm{SD}$ & $19.2 \pm 9.1$ & $11.0 \pm 5.1$ & $9.5 \pm 3.4$ & $9.9 \pm 3.6$ \\
\hline & ICC $[95 \% \mathrm{ci}]$ & $0.45[0.23-0.61]$ & $0.53[0.35-0.67]$ & $0.60[0.45-0.72]$ & $0.61[0.47-0.73]$ \\
\hline \multirow[t]{5}{*}{ Hor GRF (N) } & factor & 4 & 2 & 1 & 3 \\
\hline & correlation & 0.93 & 0.93 & 0.91 & 0.92 \\
\hline & window & $0.4-2.4 \mathrm{~s}$ & $3.0-5.0 \mathrm{~s}$ & $5.3-8.3 \mathrm{~s}$ & $8.7-11.7 \mathrm{~s}$ \\
\hline & mean $\pm \mathrm{SD}$ & $15.4 \pm 6.6$ & $7.9 \pm 2.9$ & $7.1 \pm 1.6$ & $7.0 \pm 1.4$ \\
\hline & ICC $[95 \% \mathrm{ci}]$ & $0.57[0.40-0.70]$ & $0.66[0.52-0.76]$ & $0.71[0.60-0.80]$ & $0.67[0.55-0.77]$ \\
\hline
\end{tabular}

Table 2

shows the correlation ( $r$ ) between phases (dynamic phase (DP), late dynamic phase (LDP), static phase 1 (SP1) and static phase 2 (SP2)) for the mean resultant center of pressure speed (COP speed), mean resultant center of pressure sway (COP sway) and mean resultant horizontal ground reaction force (Hor GRF).

\begin{tabular}{llll}
\hline & COP speed & COP sway & Hor GRF \\
\hline DP vs LDP & 0.44 & 0.48 & 0.42 \\
DP vs SP1 & 0.19 & 0.41 & 0.21 \\
DP vs SP2 & 0.17 & 0.44 & 0.18 \\
LDP vs SP1 & 0.37 & 0.42 & 0.41 \\
LDP vs SP2 & 0.38 & 0.43 & 0.29 \\
SP1 vs SP2 & 0.57 & 0.47 & 0.56 \\
\hline
\end{tabular}

The highest correlations were found between the static phases (0.47-0.57) (i.e. SP1 and SP2)."

\subsection{Reliability analysis}

Fig. 3 shows the mean outcome (Figs. $3 a, c$ and e) and ICC values (Figs. $3 \mathrm{~b}, \mathrm{~d}$ and $\mathrm{f}$ ) as calculated for the different window sizes, as the window moved along the time series. The ICC values were profoundly influenced by window size and timing. A consistent pattern showed initially high ICC values for the smallest window $(0.5 \mathrm{~s})$ directly following impact of the landing, where after a sharp drop of ICC values resulted in lowest values around 1-2 s. Subsequently, for the $0.5 \mathrm{~s}$ window size, ICC values tended to be higher after $5 \mathrm{~s}$ for COP speed and COP sway, however not for Hor GRF. Furthermore, in general a larger window size resulted in higher ICC values.

The dynamic phases showed lower reliability (ICC 0.45-0.68) compared to the static phases (ICC 0.60-0.86) (Table 1 ). The ICC values were highest for COP speed, ranging from 0.63 (DP) to 0.86 (SP2), somewhat lower for Hor GRF, ranging from 0.57 (DP) to 0.71 (SP1), and lowest for COP sway, where it ranged from 0.45 (DP) to 0.61 (SP2).

\subsection{Correlation between outcome measures}

The outcome measures COP speed and Hor GRF were highly inter-correlated $(r>0.70)$ in each phase, with higher correlation for dynamic phases (0.86) compared to the static phases (0.71-0.73). The COP sway also showed higher correlations with COP speed and Hor GRF for dynamic phases (0.60-0.69) than for the static phases (0.36-0.46).

\section{Discussion}

Our main finding is that the time series following a single leg drop jump landing consists of four distinct phases with unique information. These phases can be classified as dynamic $(0.4-2.7 \mathrm{~s})$, late dynamic (2.5-5.0 s), static 1 (5.0-8.3 s) and static 2 (8.1-11.7 s). Across these phases, the highest reliability was found for the COP speed (ICC ranging from 0.63 for the DP to 0.86 for the SP2). The present results are in agreement with previous studies that showed lower correlation of similar outcome measures after a jump landing task compared to a static single leg stance task $[8,25]$.

The Factor Analysis identified four patterns of variation (factors) along the time series, which represented the four phases. As each phase was highly correlated with only one factor, it is safe to assume that the four phases truly hold unique information. The results indicated that the four phases were best represented by a window size of 2 or $3 \mathrm{~s}$. As these window sizes showed distinct and very high $(r>0.90)$ peak correlations with each of the four factors, these will include most of the available information following a single leg drop jump landing. On the other hand, it still needs to be clarified whether the four phases are truly caused by differences in sensorimotor function. For instance, the two static phases revealed similar outcome values (Table 1 ), indicating that a static situation of balance was reached after $5 \mathrm{~s}$. However, both phases showed additive explanation of the variance between subjects, which might be related to the focus of attention, rather than to balance ability.

Based on the current results, future research should explore the additive value of the dynamic, late dynamic and static phase to detect impairments and predict injury risk. This may increase sensitivity and allow for better prevention and rehabilitation management.

The dynamic phases showed lower reliability (ICC $0.45-0.68$ ) compared to the static phases (ICC 0.60-0.86) (Table 1, and Figs. 3b, $\mathrm{d}$ and $\mathrm{f}$ ). The relatively low reliability may limit sensitivity to detect differences. On the other hand, despite lower reliability, outcomes of the dynamic phase may still detect impairments, due to higher effect sizes [6]. This is illustrated by the findings of Wikstrom et al., who showed that the mediolateral stability index had higher accuracy to differentiate between 'copers' and individuals with chronic ankle instability than the other stability indices, whilst having a 'poor' reliability [12,34].

In accordance with previous studies [26], COP speed showed consistently higher reliability compared to COP sway and Hor GRF. 

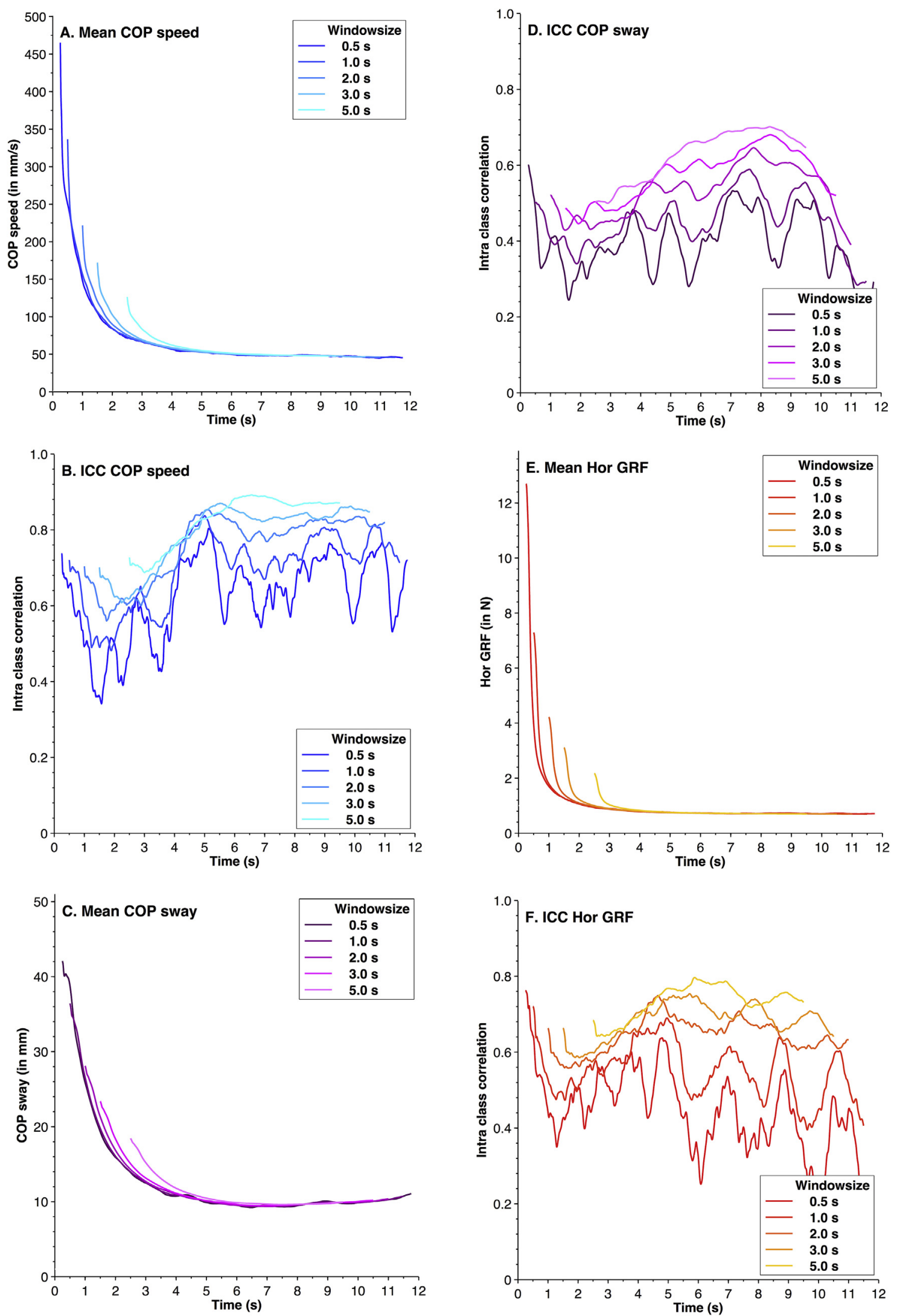

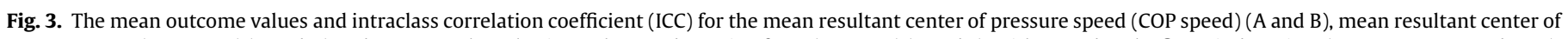

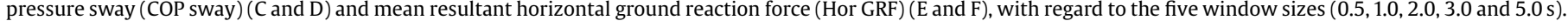
The starting point for each window size is half the length of the window, in order to facilitate comparison. 
Table 3

shows the correlation $(r)$ between the mean resultant center of pressure speed (COP speed), mean resultant center of pressure sway (COP sway) and mean resultant horizontal ground reaction force (Hor GRF) for each of the identified phases (dynamic phase (DP), late dynamic phase (LDP), static phase 1 (SP1) and static phase 2 (SP2)).

\begin{tabular}{lllll}
\hline & DP & LDP & SP1 & SP2 \\
\hline COP speed vs COP sway & 0.60 & 0.63 & 0.40 & 0.41 \\
COP speed vs Hor GRF & 0.86 & 0.86 & 0.73 & 0.71 \\
COP sway vs Hor GRF & 0.69 & 0.68 & 0.46 & 0.36 \\
\hline
\end{tabular}

The very high correlations between COP speed and Hor GRF (0.86) in the dynamic phases underline the validity of both measures for the dynamic phases (Table 3). Furthermore, it suggests that the lower reliability in the dynamic domain of both outcome measures is due to real variability of balance performance, rather than to error of measurement.

We studied a group of elite youth soccer players who practice and compete at the highest standard of competitive soccer. Therefore, the presented window selection may not be generalizable to other populations. In addition, the jump landing test is subject to methodological variation, which can alter the degree of difficulty [9]. Therefore, exact application of the presented windows may not be appropriate for other dynamic tests.

Furthermore, we opted to group both legs together for the statistical analyses. This was done since static and dynamic balance is assumed to reflect, to a large extent, total body sensorimotor function [4]. Therefore, combining both legs may improve the outcome estimate, as it increases number of measurements and thereby the reliability of whole body sensorimotor function estimates. However, it has been argued that injury risk may not be fully bilateral, especially with regard to recurrence of injury [35]. To verify that pooling of the left and right leg for the present analysis is justified, we performed a separate analysis on the left and right legs (three trials per leg). These analyses showed comparable results (see Supplementary data 2) to those reported in Table 1. However, probably as a consequence of the reduced number of repetitions per subject, the ICC values were lower in most cases. Clearly, if unilateral testing is deemed necessary, as in case of follow-up after a unilateral injury, more repetitions per leg are needed to achieve similar reliability.

Finally, we did not include the vertical GRF in our outcome measures to avoid disproportionately interference of the vertical impact of the landing on outcome values for the 'Hor GRF'. However, vertical impact forces have shown potentially independent relevance in injury assessment [9]. Therefore, in addition to the current four phases following landing, we advise to include impact forces when clinical relevance of the jump landing task is evaluated.

In conclusion, for elite youth soccer players, in a single leg drop jump landing, four distinct phases in time, the dynamic phase, late dynamic phase, and two static phases provide unique information. Reliability was higher in the static phases compared to dynamic phases, with the best reliability for COP speed.

\section{Conflict of interest statement}

There is no conflict of interest.

\section{Acknowledgments}

None.

\section{Appendix A. Supplementary data}

Supplementary data associated with this article can be found, in the online version, at http://dx.doi.org/10.1016/j. gaitpost.2016.09.002.

\section{References}

[1] C. Hrysomallis, Balance ability and athletic performance, Sports Med. 41 (2011) $221-232$.

[2] H. Kiers, S. Brumagne, J.H. van Dieën, P. van der Wees, L. Vanhees, Ankle proprioception is not targeted by exercises on an unstable surface, Eur. J. Appl. Physiol. 112 (2012) 1577-1585.

[3] M.D. Jakobsen, E. Sundstrup, P. Krustrup, P. Aagaard, The effect of recreational soccer training and running on postural balance in untrained men, Eur. J. Appl. Physiol. 111 (2011) 521-530.

[4] J. Witchalls, P. Blanch, G. Waddington, R. Adams, Intrinsic functional deficits associated with increased risk of ankle injuries: a systematic review with meta-analysis, Br. J. Sports Med. 46 (2012) 515-523.

[5] H. Negahban, M. Mazaheri, I. Kingma, J.H. van Dieen, A systematic review of postural control during single-leg stance in patients with untreated anterior cruciate ligament injury, Knee Surg. Sports Traumatol. Arthrosc. 22 (2014) 1491-1504.

[6] J.M. Dallinga, H.T.D. van der Does, A. Benjaminse, K.A.P.M. Lemmink, Dynamic postural stability differences between male and female players with and without ankle sprain, Phys. Ther. Sport 17 (2016) 69-75.

[7] M.D.W. Hupperets, E.A.L.M. Verhagen, W. van Mechelen, Effect of sensorimotor training on morphological, neurophysiological and functional characteristics of the ankle: a critical review, Sports Med. 39 (2009) 591-605.

[8] T.C. Sell, An examination, correlation, and comparison of static and dynamic measures of portural stability in healthy, physically active adults, Phys. Ther. Sport. 13 (2012) 80-86.

[9] D.P. Fransz, A. Huurnink, I. Kingma, E.A.L.M. Verhagen, J.H. van Dieën, A systematic review and meta-analysis of dynamic tests and related force plate parameters used to evaluate neuromusculoskeletal function in foot and ankle pathology, Clin. Biomech. (Bristol, Avon) 28 (2013) 591-601.

[10] D.P. Fransz, A. Huurnink, V.A. de Boode, I. Kingma, J.H. van Dieën, Time to stabilization in single leg drop jump landings: an examination of calculation methods and assessment of differences in sample rate, filter settings and trial length on outcome values, Gait Posture 41 (2015) 63-69.

[11] D.P. Fransz, A. Huurnink, V.A. de Boode, I. Kingma, J.H. van Dieën, The effect of the stability threshold on time to stabilization and its reliability following a single leg drop jump landing, J. Biomech. 49 (2016) 496-501.

[12] E.A. Wikstrom, M.D. Tillman, A.N. Smith, P.A. Borsa, A new force-plate technology measure of dynamic postural stability: the dynamic postural stability index, J. Athl. Training 40 (2005) 305-309.

[13] K. Liu, J. Glutting, E.A. Wikstrom, G. Gustavsen, T. Royer, T.W. Kaminski, Examining the diagnostic accuracy of dynamic postural stability measures in differentiating among ankle instability status, Clin. Biomech. (Bristol, Avon) 28 (2013) 211-217.

[14] C.N. Brown, S.E. Ross, R. Mynark, K.M. Guskiewicz, Assessing functional ankle instability with joint position sense, time to stabilization, and electromyography, J. Sport Rehabil. 13 (2004) 122-134.

[15] S.E. Ross, K.M. Guskiewicz, M.T. Gross, B. Yu, Balance measures for discriminating between functionally unstable and stable ankles, Med. Sc.i Sports Exerc. 41 (2009) 399-407.

[16] S.E. Ross, K.M. Guskiewicz, B. Yu, Single-leg jump-landing stabilization times in subjects with functionally unstable ankles, J. Athl. Train 40 (2005) 298-304.

[17] S.M. Colby, R.A. Hintermeister, M.R. Torry, J.R. Steadman, Lower limb stability with ACL impairment, J. Orthop. Sports Phys. Ther. 29 (1999) 444-451.

[18] K.A. Webster, P.A. Gribble, Time to stabilization of anterior cruciate ligamentreconstructed versus healthy knees in National Collegiate Athletic Association Division I female athletes, J. Athl. Train 45 (2010) 580-585.

[19] R. De Ridder, T.M. Willems, J. Vanrenterghem, P. Roosen, Effect of a homebased balance training protocol on dynamic postural control in subjects with chronic ankle instability, Int. J. Sports Med. 36 (2015) 596-602.

[20] P.A. Gribble, B.L. Taylor, J. Shinohara, Bracing does not improve dynamic stability in chronic ankle instability subjects, Phys. Ther. Sport 11 (2010) 3-7.

[21] T. Paillard, F. Noé, T. Rivière, V. Marion, R. Montoya, P. Dupui, Postural performance and strategy in the unipedal stance of soccer players at different levels of competition, J. Athl. Train 41 (2006) 172-176.

[22] E.A. Wikstrom, K.A. Fournier, P.O. McKeon, Postural control differs between those with and without chronic ankle instability, Gait Posture 32 (2010) 82-86.

[23] M.A. Hoffman, D.M. Koceja, Dynamic balance testing with electrically evoked perturbation: a test of reliability, Arch. Phys. Med. Rehabil. 78 (1997) 290-293.

[24] C. Hrysomallis, P. McLaughlin, C. Goodman, Relationship between static and dynamic balance tests among Australian footballers, J. Sci. Med. Sport 9 (2006) 288-291.

[25] M. Pau, F. Arippa, B. Leban, F. Corona, G. Ibba, F. Todde, et al., Relationship between static and dynamic balance abilities in Italian professional and youth league soccer players, Phys. Ther. Sport 16 (2015) 236-241.

[26] D.P. Fransz, A. Huurnink, I. Kingma, J.H. van Dieën, How does postural stability following a single leg drop jump landing task relate to postural stability during a single leg stance balance task, J. Biomech. 47 (2014) 3248-3253. 
[27] A. Huurnink, D.P. Fransz, I. Kingma, J.H. van Dieën, Comparison of a laboratory grade force platform with a Nintendo Wii Balance Board on measurement of postural control in single-leg stance balance tasks, J. Biomech. 46 (2013) 13921395.

[28] R.J. Doyle, E.T. Hsiao-Wecksler, B.G. Ragan, K.S. Rosengren, Generalizability of center of pressure measures of quiet standing, Gait Posture 25 (2007) 166-171.

[29] A. Huurnink, D.P. Fransz, I. Kingma, E.A.L.M. Verhagen, J.H. van Dieën, Postural stability and ankle sprain history in athletes compared to uninjured controls, Clin. Biomech. (Bristol, Avon) 29 (2014) 183-188.

[30] M. Salavati, M.R. Hadian, M. Mazaheri, H. Negahban, I. Ebrahimi, S. Talebian, et al., Test-retest reliability of center of pressure measures of postural stability during quiet standing in a group with musculoskeletal disorders consisting of low back pain, anterior cruciate ligament injury and functional ankle instability, Gait Posture 29 (2009) 460-464.
[31] R.A. Clark, A.L. Bryant, Y. Pua, P. McCrory, K. Bennell, M. Hunt, Validity and reliability of the Nintendo Wii Balance Board for assessment of standing balance, Gait Posture 31 (2010) 307-310.

[32] I.T. Jolliffe, Principal Component Analysis, second ed., Springer, New York, 2002.

[33] H.C.W. de Vet, C.B. Terwee, D.L. Knol, L.M. Bouter, When to use agreement versus reliability measures, J. Clin. Epidemiol. 59 (2006) 1033-1039.

[34] E.A. Wikstrom, M.D. Tillman, T.L. Chmielewski, J.H. Cauraugh, K.E. Naugle, P.A Borsa, Discriminating between copers and people with chronic ankle instability, J. Athl. Train 47 (2012) 136-142.

[35] M.V. Paterno, L.C. Schmitt, K.R. Ford, M.J. Rauh, G.D. Myer, B. Huang, et al., Biomechanical measures during landing and postural stability predict second anterior cruciate ligament injury after anterior cruciate ligament reconstruction and return to sport, Am. J. Sports Med. 38 (2010) 1968-1978. 